\title{
Evidências sobre tratamentos clínicos conservadores para doença hemorroidária
}

\author{
Evidence on conservative clinical treatments for haemorrhoids

\section{Evidencias sobre tratamientos clínicos conservadores para la enfermedad hemorroidal}

Fernanda da Silva Barbosa. Universidade Federal de Santa Catarina (UFSC). Florianópolis, SC, Brasil. fernandinhasbarbosa@hotmail.com (Autora correspondente) Jardel Corrêa de Oliveira. Secretaria Municipal de Saúde (SMS). Florianópolis, SC, Brasil. jardel_coli@yahoo.com.br

Charles Dalcanale Tesser. Universidade Federal de Santa Catarina (UFSC). Florianópolis, SC, Brasil. charlestesser@ccs.ufsc.br

\section{Resumo}

Objetivo: 0 objetivo desta avaliação de tecnologia em saúde foi analisar as evidências sobre tratamentos clínicos conservadores para doença hemorroidária utilizáveis na Atenção Primária à Saúde. Métodos: buscou-se no Embase, LILACS e MEDLINE via Pubmed por meta-análises, revisões sistemáticas e ensaios clínicos controlados e aleatorizados, publicados até dezembro de 2012, sem limite de linguagem. Os estudos deveriam avaliar os efeitos dos tratamentos clínicos conservadores (fibras ou laxantes, flavonoides, analgésicos, corticosteroides, banhos de assento ou pomadas de nitroglicerina) comparados a placebo ou entre si. Os desfechos considerados foram: melhora global dos sintomas, sangramento, prurido, dor, prolapso e efeitos adversos. Resultados: uma meta-análise demonstrou que fibras promovem melhora global dos sintomas e do sangramento e diminuem a recorrência após procedimentos ambulatoriais. Três meta-análises mostraram a eficácia de flavonoides para sangramento agudo e pós-operatório, melhora global dos sintomas, exsudação perianal e recorrência após episódio agudo. Não houve diferença estatística para prurido, dor, prolapso ou efeitos adversos nos dois casos. Flavonoides do tipo rutosídeos reduziram sintomas em gestantes, apesar da insuficiência dos dados para comprovar sua segurança. Não foram encontrados estudos que preenchessem os critérios de seleção para os demais tipos de tratamento. Conclusões: fibras ou flavonoides orais podem ser utilizados na atenção primária para a melhora global dos sintomas e sangramento na doença hemorroidária graus I e ll; para paciente grau III que não deseje se submeter a procedimento ambulatorial; e no pós-operatório. Ensaios clínicos controlados e aleatorizados com qualidade metodológica adequada são necessários para confirmar estes resultados.

\section{Abstract}

Objective: The objective of this health technology assessment was to analyse the evidence on conservative clinical treatments for haemorrhoids usable in Primary Health Care. Methods: We searched in Embase, LILACS and MEDLINE through Pubmed for meta-analyses, systematic reviews and randomized controlled trials published until December 2012, without limits of language. Studies should evaluate the effects of conservative medical treatments (fibres or laxatives, flavonoids, analgesics, corticosteroids, sitz baths or nitro-glycerine ointments) compared to placebo or each other. The outcomes considered were overall symptom improvement, bleeding, itching, pain, prolapse and adverse effects. Results: One meta-analysis showed that fibres promote overall improvement of symptoms and bleeding; and decrease recurrence after outpatient procedures. Three meta-analyses showed efficacy of flavonoids for acute and postoperative bleeding, overall symptom improvement, perianal discharge and recurrence after acute episode. There was no statistical difference for itching, pain, prolapse, or adverse effects in both cases. Rutosides, a type of flavonoid, reduced symptoms in pregnant women, despite the insufficiency of data to prove its safety. No studies were found on other types of treatment that met the selection criteria. Conclusions: In Primary Health Care, oral fibres or flavonoids can be used to improve overall symptoms and bleeding in haemorrhoid patients at grades I and II; to patient grade III who does not wish to undergo outpatient procedure; and postoperatively. Randomized controlled trials with adequate methodological quality are needed to confirm these results.

\section{Palavras-chave:}

Avaliação da Tecnologia

Biomédica

Terapêutica

Hemorroidas

Atenção Primária à Saúde

\section{Keywords:}

Technology Assessment, Biomedical

Therapeutics Hemorrhoids Primary Health Care
Fonte de financiamento: declaram não haver.

Parecer CEP:

não se aplica.

Conflito de interesses: declaram não haver.

Recebido em: 28/07/2013. Aprovado em: 16/12/2013. 


\section{Resumen}

Objetivo: el objetivo de esta evaluación de tecnología sanitaria es analizar la evidencia sobre tratamientos clínicos conservadores para las hemorroides utilizables en la atención primaria de la salud. Métodos: se buscó en Embase, LILACS y MEDLINE a través de Pubmed por metanálisis, revisiones sistemáticas, y ensayos clínicos controlados y aleatorizados publicados hasta diciembre de 2012, en todos los idiomas. Los estudios deberían evaluar los efectos de los tratamientos clínicos conservadores (fibras o laxantes, flavonoides, analgésicos, cortico esteroides, baños de asiento o ungüentos de nitroglicerina) comparándolos con placebo o entre sí. Los resultados que se consideraron fueron: mejora general de los síntomas, sangrado, picazón, dolor, prolapso y efectos adversos. Resultados: un metanálisis demostró que las fibras promueven una mejora general de los síntomas y del sangrado, y disminuyen la recaída tras los procedimientos ambulatorios. Tres metanálisis mostraron la eficacia de los flavonoides para la hemorragia aguda y el postoperatorio, mejora general de los síntomas, exudación perianal y recaída después del episodio agudo. No hubo diferencia estadística para picazón, dolor, prolapso, o efectos adversos en ambos casos. Los flavonoides del tipo rutosídeos redujeron los síntomas en las gestantes, a pesar de la falta de datos para demostrar su seguridad. No se encontraron estudios que cumplieran con los criterios de selección para otros tipos de tratamiento. Conclusiones: las fibras o flavonoides orales pueden ser utilizados en la atención primaria para una mejora global de los síntomas y del sangrado en las hemorroides de grado I y II; en paciente grado III que no quiera someterse a procedimiento ambulatorio; y en el postoperatorio. Se necesitan ensayos clínicos controlados y aleatorizados con una calidad metodológica adecuada para confirmar estos resultados.

\section{Palabras clave:}

Evaluación de la Tecnología

Biomédica

Terapéutica

Hemorroides

Atención Primaria de Salud

\section{Introdução}

Hemorroidas são estruturas vasculares presentes na região anorretal constituídas por arteríolas e vênulas. De acordo com a localização, dividem-se em internas (acima da linha pectínea, no espaço submucoso) e externas (abaixo da linha pectínea, no espaço subcutâneo).$^{1-3}$ Em conjunto com o sistema neuromuscular do ânus, formado por esfíncteres, nervos, mucosa e pele, as hemorroidas contribuem para a manutenção da continência fecal e diferenciação entre a natureza do conteúdo no interior do canal anal. ${ }^{4,5}$ Quando há alteraçóes estruturais e vasculares, associadas a hábitos dietéticos ou defecatórios errôneos, as hemorroidas podem tornar-se sintomáticas, caracterizando a doença hemorroidária. ${ }^{1,4}$

É uma das afecçôes coloproctológicas mais frequentes e sua importância já era descrita por Hipócrates (460 a.C.) na Grécia antiga. ${ }^{1,6} \mathrm{~A}$ real prevalência da doença hemorroidária na atualidade é controversa, podendo se dar por subnotificação dos sintomas por parte dos pacientes ou pela atribuição de quaisquer males anorretais a esta doença. ${ }^{6,7}$ Estima-se que $4,4 \%$ da populaçáo geral nos EUA seja portadora de doença hemorroidária, sendo que apenas um terço procura por atendimento médico. Incide igualmente em ambos os sexos e tem um pico de prevalência entre 45-65 anos. ${ }^{8,9}$ No Brasil, um estudo sugere uma incidência de 27,32\% em pacientes atendidos pelo serviço de coloproctologia, com predominância no sexo feminino. ${ }^{6}$

Os sintomas mais comuns são sangramento, prolapso, prurido, dor ou desconforto anal e, menos frequentemente, exsudação perianal. ${ }^{1,6,7,10}$ A doença hemorroidária, em sua classificação mais utilizada, pode ser interna, externa ou mista, de acordo com a localizaçáo do mamilo hemorroidário no canal anal, conforme descrito previamente. A fim de guiar a terapêutica mais adequada, Banov et al. ${ }^{11}$ propuseram classificar a forma interna com base no grau de prolapso e sangramento em a) Grau I: quando há sangramento, porém não prolaba abaixo da linha pectínea aos esforços ou evacuação; b) Grau II: quando prolaba abaixo da linha pectínea, ao esforço evacuatório, com prolapso pelo ânus, porém retraindo espontaneamente após cessado o esforço; c) Grau III: quando prolaba à evacuação e/ou aos esforços, com prolapso pelo ânus, porém sem retração espontânea, sendo necessário redução digital; e d) Grau IV: quando o prolapso é irredutível e o mamilo hemorroidário permanece permanentemente prolabado. ${ }^{1,7,12}$

O manejo se faz de acordo com a gravidade dos sintomas, presença ou náo de complicaçóes e o grau da hemorroida interna. Segundo o UpToDate, Prodigy, Current e os protocolos disponíveis no National Guideline Clearinghouse, a abordagem inicial dos casos não complicados e principalmente graus I e II consiste em medidas conservadoras como uso de fibras ou laxantes na dieta, medicamentos flebotônicos como flavonoides, analgésicos orais ou tópicos, corticosteroides tópicos, banhos de assento e pomadas à base de nitroglicerina. Na doença de graus I, II e III sem resposta ao tratamento conservador, o tratamento consiste de procedimentos ambulatoriais não cirúrgicos como escleroterapia, crioterapia, fotocoagulação e ligadura elástica. Somente de $5 \%$ a $10 \%$ dos casos, principalmente aqueles com hemorroidas internas grau IV ou que não obtiveram sucesso com outras terapias, necessitam de tratamento cirúrgico, a hemorroidectomia. 1,2,3,7,8,10-19

No contexto do Sistema Único de Saúde (SUS), a abordagem abrange o princípio da hierarquização, pois pode necessitar de atendimento em diferentes níveis de atenção, dependendo da resposta aos tratamentos e do grau da doença. A Atenção Primária à Saúde (APS) representa a porta de entrada do sistema, responsável pela ordenação e continuidade do cuidado da população vinculada a uma unidade básica de saúde, definida com base territorial. Nela realizam-se muitas vezes o diagnóstico e a primeira abordagem de tratamento, que consiste nas medidas clínicas conservadoras. Casos que não melhoram com estas medidas ou dependendo do grau da doença podem necessitar de encaminhamento para a atenção secundária ou terciária para 
a especialidade de coloproctologia. Os procedimentos ambulatoriais não cirúrgicos descritos anteriormente são realizados na atenção secundária. A atenção terciária compreende o atendimento no ambiente hospitalar, no qual são realizadas as cirurgias. No período pós-procedimento ambulatorial ou cirúrgico, a pessoa retorna para acompanhamento na APS..$^{20-25}$

A grande maioria das pessoas com doença hemorroidária beneficiar-se-á apenas de tratamentos clínicos conservadores, que podem ser realizados de forma eficaz e segura pelo médico de família, sem necessidade de maiores intervençóes. No entanto, ainda há inconsistência sobre quais terapias devem ser utilizadas no paciente da atenção primária. O objetivo desta revisão foi avaliar as evidências sobre tratamentos clínicos conservadores para doença hemorroidária que podem ser realizados na APS.

\section{Métodos}

Realizou-se um estudo de Avaliação de Tecnologia em Saúde (ATS) dos tratamentos clínicos conservadores utilizados na doença hemorroidária no âmbito da APS, conforme diretrizes metodológicas para elaboração de pareceres técnico-científicos padronizadas pelo Ministério da Saúde. ${ }^{26}$ Com base na literatura, foram selecionados como objetos de estudo os seguintes tratamentos: fibras ou laxantes (sendo os mais citados: Psyllium, metilcelulose, Sterculia, docusato de sódio, lactulose e Senna), flavonoides (dentre eles: rutosídeo, diosmina e hesperidina), analgésicos orais e tópicos (lidocaína e cinchocaína), corticosteroides tópicos (sendo hidrocortisona e fluocortolona os mais comuns), banhos de assento e pomadas à base de nitroglicerina. ${ }^{15-19}$

De acordo com o modelo PICO (População; Intervenção; Controle; Outcome ou Desfecho), ${ }^{27}$ buscou-se por estudos que avaliaram o uso de tais tratamentos comparados entre si ou a placebo, em pessoas com doença hemorroidária. Foram considerados como desfechos: melhora global, sangramento, dor ou irritação, prurido, prolapso e efeitos adversos. Foram obtidos, por meio do MeSH e DeCS, diversos descritores para doença hemorroidária para cada um dos tipos de tratamento e termos correlatos.

Inicialmente realizou-se uma busca por meta-análises (MA) e revisóes sistemáticas (RS) no MEDLINE via Pubmed, Embase e LILACS, para cada uma das formas de tratamento separadamente. Não houve restriçáo de linguagem, sendo os limites: estudos publicados até dezembro de 2012, humanos e tipo de estudo. As estratégias de busca e todos os descritores utilizados são descritos no Quadro 1.

Quadro 1. Estratégias de busca da pesquisa.

Termos

Hemorroidas e Fibras/ Laxantes/

Psyllium/ Metilcelulose/ Sterculia/

Docusato de Sódio/ Lactulose/ Senna

(Hemorrhoids or Haemorrhoids) AND ("Fiber, Dietary" OR "Dietary Fibers" OR "Fibers, Dietary" OR "Wheat Bran" OR "Bran, Wheat” OR "Brans, Wheat" OR "Wheat Brans" OR Roughage OR Roughages OR Laxatives OR Cathartics OR "Bulk Laxatives" OR "Laxatives, Bulk" OR Psyllium OR "Ispaghule Gum" OR "Gum, Ispaghule" OR "Plantago Seed" OR "Seed, Plantago" OR Ispaghula OR Methylcellulose OR "Methyl Cellulose" OR "Cellulose, Methyl" OR Sterculia OR Sterculias OR Karaya OR Karayas OR "Sterculia urens" OR "Sterculia uren" OR "uren, Sterculia" OR "urens, Sterculia” OR Lactulose OR "Dioctyl Sulfosuccinic Acid” OR "Dioctyl Sulfosuccinate" OR DOSS OR Dioctylsulfosuccinate OR "Dioctyl Sulfosuccinates" OR "Sulfosuccinates, Dioctyl” OR "Sodium Dioctylsulphosuccinate" OR "Dioctylsulphosuccinate, Sodium" OR "Sodium Dioctyl Sulfosuccinate” OR “Dioctyl Sulfosuccinate, Sodium” OR "Docusate Sodium” OR "Sodium, Docusate" OR "Senna Extract" OR "Extract, Senna")

Hemorroidas e Flavonoides/ Hidroxietil (Hemorrhoids OR Haemorrhoids) AND (Flavonoids OR Bioflavonoids OR Hydroxyethylrutoside OR Diosmin OR "Buchu Resin" OR Rutosídeos/ Diosmina/ Hesperidina Hemorroidas e Analgésicos/ Lidocaína/ Chinchocaína

Hemorroidas e Corticosteroides/ Corticoides/ Hidrocortisona/ Fluocortolona Hemorroidas e Banho de Assento Hemorroidas e Nitroglicerina
"Resin, Buchu" OR Hesperidin) (Hemorrhoids OR Haemorrhoids) AND (Analgesics OR "Analgesic Drugs" OR "Drugs, Analgesic" OR Anodynes OR "Analgesic Agents" OR "Agents, Analgesic" OR "Antinociceptive Agents" OR "Anesthestics, local” OR "Local anesthetics" OR "anesthetics, topical” OR Lidocaine OR Lignocaine OR "2-(Diethylamino)-N-(2,6-Dimethylphenyl)Acetamide" OR “Lidocaine Carbonate (2:1)" OR "Lidocaine Carbonate" OR "Carbonate, Lidocaine" OR “Lidocaine Hydrocarbonate" OR "Hydrocarbonate, Lidocaine" OR "Lidocaine Hydrochloride" OR "Hydrochloride, Lidocaine" OR " Lidocaine Monohydrochloride" OR "Monohydrochloride, Lidocaine" OR "Lidocaine Monoacetate" OR "Monoacetate, Lidocaine" OR "Lidocaine Sulfate (1:1)" OR Octocaine OR Xylesthesin OR Xylocaine OR "Xylocaine CO2" OR "Lidocaine Monohydrochloride, Monohydrate" OR "Monohydrate Lidocaine Monohydrochloride" OR Dibucaine OR Cinchocaine OR Sovcaine OR Nupercaine OR Cincain OR Nupercainal) (Hemorrhoids OR Haemorrhoids) AND (corticosteroids OR corticoids OR hydrocortisone OR cortisol OR "Hydrocortisone, (9 beta, 10 alpha, 11 alpha)-Isomer" OR "Hydrocortisone, (11 alpha)-Isomer" OR fluocortolone OR "Fluocortolone Pivalate" OR "Pivalate, Fluocortolone" OR "Fluocortolone Caproate" OR "Caproate, Fluocortolone") (Hemorrhoids OR Haemorrhoids) AND ("Sitz bath" OR "warm-water bath") (Hemorrhoids OR Haemorrhoids) AND (Nitroglycerin OR "Glyceryl Trinitrate" OR "Trinitrate, Glyceryl") 
Os estudos identificados em cada base de dados foram agrupados no software de manejo de referências EndNote versão X5 de acordo com a forma de tratamento (cada uma das estratégias de busca descritas no Quadro 1), sendo excluídos os duplicados. Os critérios de seleção para esta busca incluíram: desenho dos estudos (MA ou RS), população (pessoas com hemorroidas) e intervenção (tratamento clínico conservador versus placebo ou outro tratamento clínico conservador). Foram excluídos estudos com outro tipo de desenho que não MA ou RS, população composta por animais, intervenção diferente daquela considerada na estratégia e ainda estudos sobre outras doenças ou temas.

O resultado da busca foi avaliado de forma independente por dois dos autores deste estudo de avaliação de tecnologia em saúde, de acordo com o título e resumo e quanto ao preenchimento dos critérios de seleção. Quando o título ou o resumo não permitiam a exclusão e o estudo estava disponível na íntegra, a avaliação foi feita com base na metodologia do artigo. Quando o texto completo não estava disponível, tentou-se obtê-lo por meio de contato com professores de Universidades da Espanha e Canadá, sendo então excluídos os estudos que não foram obtidos. A Tabela 1 demonstra de forma detalhada o processo de seleção.

Tabela 1. Seleção das revisões sistemáticas e meta-análises.

\begin{tabular}{|c|c|c|c|c|c|c|}
\hline \multirow[t]{2}{*}{ Tratamento } & \multirow[t]{2}{*}{ Estudos identificados } & \multicolumn{4}{|c|}{$\begin{array}{l}\text { Estudos que não preencheram critérios de seleção após análise do título e/ou do resumo ou } \\
\qquad \text { da metodologia }\end{array}$} & \multirow[t]{2}{*}{$\begin{array}{c}\text { Estudos } \\
\text { Selecionados }\end{array}$} \\
\hline & & $\begin{array}{l}\text { Outros desenhos de } \\
\text { estudo }\end{array}$ & $\begin{array}{l}\text { Outros tipos de } \\
\text { tratamento ou } \\
\text { medicamento } \\
\text { utilizados }\end{array}$ & $\begin{array}{l}\text { Outra doença ou } \\
\text { tema estudado }\end{array}$ & $\begin{array}{l}\text { Estudos cujo resumo } \\
\text { e/ou texto completo } \\
\text { não estavam } \\
\text { disponíveis }\end{array}$ & \\
\hline Fibras / Laxantes & $n=9$ & $n=3$ & $n=2$ & $n=3$ & $\mathrm{n}=0$ & $n=1$ \\
\hline Flavonoides & $n=9$ & $n=3$ & $n=1$ & $n=0$ & $n=2$ & $n=3$ \\
\hline Analgésicos & $n=19$ & $n=2$ & $n=13$ & $n=3$ & $n=1$ & $\mathrm{n}=0$ \\
\hline Corticosteroides & $n=10$ & $n=3$ & $n=3$ & $n=3$ & $n=1$ & $\mathrm{n}=0$ \\
\hline Banho de assento & $\mathrm{n}=0$ & $\mathrm{n}=0$ & $\mathrm{n}=0$ & $\mathrm{n}=0$ & $\mathrm{n}=0$ & $\mathrm{n}=0$ \\
\hline Nitroglicerina & $n=8$ & $n=1$ & $n=5$ & $n=2$ & $\mathrm{n}=0$ & $n=0$ \\
\hline TOTAL & $\mathrm{n}=55$ & $n=12$ & $\mathrm{n}=\mathbf{2 4}$ & $n=11$ & $n=4$ & $n=4$ \\
\hline
\end{tabular}

Em um segundo momento realizou-se uma busca por ensaios clínicos controlados aleatorizados (ECCA) para cada tipo de tratamento individualmente, nas mesmas bases de dados e com as mesmas estratégias utilizadas inicialmente. Não houve restrição de linguagem e os limites da pesquisa foram: estudos publicados até dezembro de 2012, humanos e tipo de estudo. Para tal busca, consideraram-se os mesmos critérios de seleção e metodologia descritos anteriormente, à exceção do desenho de estudo (desta vez ECCA) e adicionando-se como critério de exclusão o fato de já ter sido incluído em alguma das RS ou MA selecionadas ou ter avaliado um desfecho substituto não mensurável na prática clínica. O processo de seleção está demonstrado na Tabela 2.

Tabela 2. Seleção dos ensaios clínicos controlados e aleatorizados.

\begin{tabular}{|c|c|c|c|c|c|c|c|c|}
\hline \multirow[b]{2}{*}{ Tratamentos } & \multirow[b]{2}{*}{$\begin{array}{c}\text { Estudos } \\
\text { Identificados }\end{array}$} & \multicolumn{6}{|c|}{ Estudos que não preencheram critérios de seleção após análise do título e/ou resumo ou da metodologia } & \multirow[b]{2}{*}{$\begin{array}{c}\text { Estudos } \\
\text { Selecionados }\end{array}$} \\
\hline & & $\begin{array}{c}\text { Outros } \\
\text { desenhos de } \\
\text { estudo }\end{array}$ & $\begin{array}{l}\text { Outros tipos de } \\
\text { tratamentos ou } \\
\text { medicamentos } \\
\text { utilizados }\end{array}$ & $\begin{array}{c}\text { Outra doença } \\
\text { ou tema } \\
\text { estudado }\end{array}$ & $\begin{array}{l}\text { Resumo e/ou } \\
\text { texto completo } \\
\text { não estavam } \\
\text { disponíveis }\end{array}$ & $\begin{array}{c}\text { ECCR já } \\
\text { avaliados } \\
\text { nas MA } \\
\text { selecionadas }\end{array}$ & $\begin{array}{l}\text { Desfecho não } \\
\text { mensurável na } \\
\text { prática clínica }\end{array}$ & \\
\hline Fibras / Laxantes & $\mathrm{n}=47$ & $n=3$ & $\mathrm{n}=37$ & $n=3$ & $n=1$ & $n=3$ & $\mathrm{n}=0$ & $\mathrm{n}=0$ \\
\hline Flavonoides & $n=31$ & $n=2$ & $n=14$ & $n=1$ & $n=1$ & $n=13$ & $\mathrm{n}=0$ & $n=0$ \\
\hline Analgésicos & $n=175$ & $n=2$ & $n=134$ & $n=38$ & $n=0$ & $n=1$ & $\mathrm{n}=0$ & $n=0$ \\
\hline Corticosteroides & $n=21$ & $n=1$ & $n=7$ & $n=7$ & $n=4$ & $n=2$ & $n=0$ & $n=0$ \\
\hline Banhos de assento & $n=2$ & $\mathrm{n}=0$ & $n=2$ & $n=0$ & $n=0$ & $\mathrm{n}=0$ & $\mathrm{n}=0$ & $n=0$ \\
\hline Nitroglicerina & $n=19$ & $n=1$ & $n=12$ & $n=4$ & $n=1$ & $\mathrm{n}=0$ & $n=1$ & $n=0$ \\
\hline TOTAL & $n=295$ & $n=9$ & $\mathrm{n}=206$ & $n=53$ & $n=7$ & $n=19$ & $n=1$ & $\mathrm{n}=\mathbf{0}$ \\
\hline
\end{tabular}


A avaliação da qualidade dos estudos selecionados baseou-se nas diretrizes metodológicas para elaboração de pareceres técnico-científicos padronizadas pelo Ministério da Saúde. ${ }^{26}$

\section{Resultados e Discussão}

Foram identificados quatro estudos para incluir nesta ATS, cujas características estáo descritas no Quadro 2.

Quadro 2. Características dos estudos selecionados.

\begin{tabular}{|c|c|c|c|}
\hline Estudo & Tipo de Estudo e População & Intervenção & Desfechos Avaliados \\
\hline Alonso-Coello et al. ${ }^{10}$ & $\begin{array}{l}\text { - Meta-análise } \\
\text { - } 7 \text { ECCR ( } n=378) \\
\text { - Adultos com doença hemorroidária (Graus } \\
\text { I-III) }\end{array}$ & $\begin{array}{c}\text { Fibras (Ispaghula, Psyllium ou Plantago ovata, } \\
\text { Sterculia e farelo não processado) } \\
\text { versus } \\
\text { Placebo }\end{array}$ & $\begin{array}{l}\text { - Melhora Global } \\
\text { - Sangramento } \\
\text { - Prolapso } \\
\text { - Dor } \\
\text { - Prurido } \\
\text { - Recorrência ou necessidade de outros } \\
\text { tratamentos } \\
\text { - Efeitos Adversos }\end{array}$ \\
\hline Quijano e Abalos ${ }^{12}$ & $\begin{array}{l}\text { - Meta-análise } \\
\text { - } 2 \text { ECCR ( } n=150) \\
\text { - Mulheres grávidas com doença } \\
\text { hemorroidária (Graus I-III) }\end{array}$ & $\begin{array}{l}\text { Rutosídeo } \\
\text { versus } \\
\text { Placebo }\end{array}$ & $\begin{array}{l}\text { - Melhora Global } \\
\text { - Efeitos Adversos } \\
\text { - Efeitos no bebê }\end{array}$ \\
\hline Alonso-Coello et al. ${ }^{28}$ & $\begin{array}{l}\text { - Meta-análise } \\
\text { - } 14 \text { ECCR ( } n=1514) \\
\text { - Adultos com doença hemorroidária }\end{array}$ & $\begin{array}{c}\text { Flavonoides (Diosmina+hesperidina, rutosídeo, } \\
\text { troxerrutina e troxerrutina+carbazocromo) } \\
\text { versus } \\
\text { Placebo }\end{array}$ & $\begin{array}{l}\text { - Melhora Global } \\
\text { - Sangramento } \\
\text { - Dor } \\
\text { - Prurido } \\
\text { - Recorrências } \\
\text { - Efeitos Adversos }\end{array}$ \\
\hline Perera et al. ${ }^{7}$ & $\begin{array}{l}\text { - Meta-análise } \\
\text { - } 24 \text { ECCR (n=2344) } \\
\text { - Adultos com doença hemorroidária ou } \\
\text { pós-hemorroidectomia }\end{array}$ & $\begin{array}{c}\text { Flavonoides (Diosmina+hesperidina, } \\
\text { troxerrutina+carbazocromo e rutosídeo) } \\
\text { versus } \\
\text { Placebo ou outros tratamentos clínicos e } \\
\text { conservadores }\end{array}$ & $\begin{array}{l}\text { - Melhora Global } \\
\text { - Sangramento } \\
\text { - Dor } \\
\text { - Prurido } \\
\text { - Descarga ou Vazamento } \\
\text { - Consumo de analgésico } \\
\text { - Pós-operatório } \\
\text { - Efeitos Adversos }\end{array}$ \\
\hline
\end{tabular}

\section{Fibras ou laxantes}

Uma meta-análise ${ }^{10}$ que avaliou o uso de fibras e laxantes versus placebo em adultos com doença hemorroidária de grau I a III demonstrou diferença estatisticamente significante na melhora global dos sintomas e no sangramento. Foram selecionados ensaios clínicos somente com fibras do tipo agentes formadores de massa, como Plantago ovata ou Psyllium, Ispaghula husk e Sterculia. Não foram encontrados estudos com outros tipos de laxantes.

Houve redução no risco absoluto de persistência ou não melhora dos sintomas de 24\% (NNT 4; RR 0,47; IC 95\% 0,32 a 0,$68 ; I^{2}=1,1 \%$ ), e de sangramento de $26 \%$ (NNT 3; RR 0,50; IC 95\% 0,28 a 0,89; $I^{2}=45 \%$ ).

Para prolapso, dor e prurido, não houve melhora estatisticamente significante no grupo de fibras em relação ao placebo, tampouco houve diferença na incidência de efeitos adversos. Quanto ao prolapso, não surpreende a ausência de benefício com a mudança na consistência das fezes ou na frequência da defecação decorrente do uso de fibras, pois se trata de uma alteração estrutural anatômica. 
Um estudo ( $\mathrm{n}=92$ ) incluído na revisão comparou o número de recorrências em 18 meses com uso de fibras ou placebo em pacientes com doença grau III depois de submetidos à ligadura elástica. Houve redução no risco absoluto de recorrência global dos sintomas de 30\% (NNT 3; RR 0,34; IC95\% 0,15 a 0,77).

Os autores da meta-análise ${ }^{10}$ consideraram a possiblidade de viés de publicação. No entanto buscou-se minimizar essa limitação por meio de contato com autores, especialistas e farmacêuticos, tendo sido encontrados dois estudos não publicados. Os ensaios selecionados foram considerados de moderada qualidade devido a problemas no sigilo de alocaçáo, cegamento e ausência de informaçóes sobre perdas de seguimento.

\section{Flavonoides}

Foram encontradas três meta-análises, sendo uma de ensaios clínicos somente com gestantes. Nesse estudo ${ }^{12}$ avaliou-se o uso de rutosídeo por via oral versus placebo, em dois ensaios clínicos com 150 mulheres grávidas com doença hemorroidária de grau I a III, entre a décima quarta e trigésima terceira semana de gestação. Houve diferença estatisticamente significante na melhora global dos sintomas, com reduçáo no risco absoluto de persistência ou náo melhora do quadro, em quatro semanas, de 62\% (NNT 2; RR 0,07; IC95\% 0,03 a 0,20; $\mathrm{I}^{2}=0 \%$ ). Não houve diferença na incidência de efeitos adversos entre os dois grupos. Com relação aos possíveis efeitos para a criança, apesar da ocorrência de um óbito fetal no grupo controle e um caso de polidactilia no grupo dos flavonoides, não houve diferença estatisticamente significante na morte fetal, malformação congênita ou parto prematuro. Os ensaios incluídos nesse estudo foram considerados de baixa qualidade pelos autores devido a problemas no sigilo de alocação, aleatorização, descrição do controle (placebo) e tamanho das amostras.

Duas meta-análises avaliaram adultos com doença hemorroidária. O estudo de Alonso-Coello et al. ${ }^{28}$ incluiu 14 ensaios clínicos que compararam flavonoides (associação de diosmina e hesperidina, rutosídeo, troxerrutina e associação de troxerrutina com carbazocromo) versus placebo, em pacientes portadores de doença aguda, grau I a III. Já o estudo de Perera et al. ${ }^{7}$ incluiu 24 ensaios clínicos que consideraram pacientes com qualquer grau de doença hemorroidária, tanto em sua forma aguda quanto no pós-operatório de hemorroidectomia ou após ligadura elástica. Essa meta-análise comparou o uso de medicamentos flebotônicos, incluindo os mesmos avaliados por Alonso-Coello et al. ${ }^{28}$ além de um não pertencente à classe de flavonoides, versus placebo ou outros tratamentos clínicos conservadores. Perera et al.7 incluíram estudos mais recentes e consideraram o período pós-operatório, enquanto Alonso-Coello et al., ${ }^{28}$ apesar de mais antigo, selecionaram estudos que Perera et al. não incluíram, tampouco excluíram de sua meta-análise. Nos dois estudos ${ }^{7,28}$ os três tipos de flavonoides citados anteriormente foram avaliados para todos os desfechos considerados no PICO.

Para a avaliação da melhora global dos sintomas, Alonso-Coello et al. ${ }^{28}$ incluíram os quatro ensaios com flavonoides utilizados por Perera et al. ${ }^{7}$ além de outros cinco. Tal desfecho foi avaliado também em subgrupos de acordo com o período de seguimento de até sete dias, até quatro semanas e de 60 dias. Houve diferença estatisticamente significante entre os grupos, com redução no risco absoluto de persistência ou não melhora do quadro de 32,8\% (RR 0,42; IC95\% 0,28 a 0,61; $\left.\mathrm{I}^{2}=69,9 \%\right)$, que foi semelhante à encontrada nos períodos de até sete dias e até quatro semanas. Apenas um estudo ( $\left.\mathrm{n}=100\right)$ avaliou esse desfecho com seguimento de 60 dias, demonstrando uma redução ainda maior no risco de persistência ou não melhora do quadro (RR 0,17; IC95\% 0,08 a 0,37).

Os ensaios utilizados para a avaliação da dor não foram os mesmos nas duas meta-análises. Alonso-Coello et al. ${ }^{28}$ mostraram diferença estatisticamente significante entre os grupos, com redução absoluta no risco de permanecer com dor de 26,9\% (RR 0,35; IC95\% 0,18 a 0,69). Porém, os estudos foram muito heterogêneos $\left(I^{2}=84,1 \%\right)$. Perera et al. ${ }^{7}$ avaliaram esse desfecho na doença aguda e no pós-operatório, mas não houve diferença estatisticamente significante em ambos os cenários.

Para sangramento, diferentes ensaios foram avaliados nas duas meta-análises. Perera et al. ${ }^{7}$ mostraram benefício estatisticamente significante entre os grupos, tanto no sangramento agudo como no pós-operatório (OR 0,12; IC95\% 0,04 a 0,37 e OR 0,18; IC95\% 0,06 a 0,58, respectivamente, com $\mathrm{I}^{2}=0$ em ambos). Alonso-Coello et al. ${ }^{28}$ avaliaram esse desfecho também em subgrupos, de acordo com o período de seguimento de até sete dias, até quatro semanas e de 60 dias. Houve diferença estatisticamente significante entre os grupos, com redução no risco absoluto de persistência ou não melhora do sangramento de $21,7 \%$ (RR 0,33; IC95\% 0,19 a 0,57; $\left.\mathrm{I}^{2}=61,4 \%\right)$, que foi semelhante à encontrada nos períodos de até sete dias e até quatro semanas. Apenas um mesmo estudo $(\mathrm{n}=100)$ avaliou esse desfecho com seguimento de 60 dias, demonstrando uma redução ainda maior no risco de persistência ou não melhora do sangramento (RR 0,20; IC95\% 0,08 a 0,49). 
Ensaios clínicos distintos foram utilizados para avaliar o prurido nas duas meta-análises. Alonso-Coello et al. ${ }^{28}$ mostraram diferença estatisticamente significante, porém marginal entre os grupos, com redução no risco absoluto de permanecer com prurido de 35\% (RR=0,65; IC95\% 0,44 a 0,97; $\mathrm{I}^{2}=66,1 \%$ ). Perera et al. ${ }^{7}$ avaliaram prurido em episódios agudos e no pós-operatório, por meio de apenas um ensaio em cada cenário. Náo houve diferença estatisticamente significante nos casos agudos de prurido. Já no pós-operatório, houve benefício, porém marginal, no grupo dos flavonoides (OR 0,25; IC95\% $0,06$ a 0,97$)$.

Quanto aos efeitos adversos, não houve diferença entre os grupos nas duas meta-análises. ${ }^{7,28}$ Alonso-Coello et al. ${ }^{28}$ salientaram que a maioria dos ensaios teve um período de seguimento menor do que três meses.

Perera et al. ${ }^{7}$ ainda avaliaram exsudação perianal, demonstrando beneficio estatisticamente significante dos flavonoides em relação ao placebo (OR 0,12; IC95\% 0,04 a 0,42 e I²=0\%). Também mensuraram a não adesão ao tratamento e não houve diferença estatística entre os grupos.

Alonso-Coello et al. ${ }^{28}$ ainda avaliaram a recorrência após um episódio agudo em um período de dois a seis meses, mostrando benefício estatisticamente significante do grupo flavonoides em relação ao placebo, com reduçáo no risco absoluto de recorrência dos sintomas de 17,2\% (RR 0,53; IC95\% 0,41 a 0,69; $\mathrm{I}^{2}=0 \%$ ).

Como limitaçôes, Alonso-Coello et al. ${ }^{28}$ consideraram a qualidade moderada dos estudos, principalmente pela ausência de informaçôes claras sobre sigilo de alocação, aleatorização e cegamento pelos autores dos ensaios, além de potencial viés de publicação, apesar dos esforços para localizar estudos não publicados. Para explicar a variabilidade entre os estudos, foi considerada a severidade da doença (maior efeito nos casos de graus I e II do que nos de graus III e IV), tipos de intervençôes diferentes (com associação a outros tratamentos clínicos conservadores ou isoladas), metodologia inadequada dos estudos e períodos de seguimento distintos. A variação na dose dos medicamentos não alterou os desfechos. Dos 14 ensaios incluídos apenas três avaliaram os resultados por intenção de tratar. Os demais o fizeram conforme protocolo ou náo deixaram clara tal informação.

Os estudos selecionados por Perera et al..$^{7}$ foram considerados de moderada qualidade, uma vez que alguns realizaram inadequados cegamentos ou sigilo de alocação e outros não trouxeram informaçôes claras sobre desfechos, seguimento ou financiamento do estudo. Houve dificuldade de reunir e comparar os dados dos ensaios, visto que muitos deles utilizaram parâmetros subjetivos, como escalas de sintomas, e outras variáveis dicotômicas para avaliar os desfechos. As diferentes doses utilizadas dos medicamentos e a localização geográfica dos estudos (com dietas e estilos de vida que variam da Ásia para a Europa) são possíveis explicaçóes para a heterogeneidade entre os ensaios.

\section{Analgésicos, corticosteroides e banhos de assento}

Não foram encontrados estudos que preenchessem os critérios de seleção para o tratamento da doença hemorroidária com analgésicos orais ou tópicos, corticosteroides tópicos e banhos de assento.

\section{Nitroglicerina}

Foi encontrado um $\mathrm{ECCA}^{29}$ para o tratamento da doença hemorroidária com pomada à base de nitroglicerina. No entanto, este media como desfecho a diminuição da pressão do esfíncter anal interno por meio de manometria retal de 24 horas. Tal estudo foi excluído por considerar apenas um desfecho náo mensurável na prática clínica, não preenchendo os critérios de seleção.

\section{Contextualização ao cenário de prática da APS e Medicina de Família no Brasil}

A doença hemorroidária é uma afecção comum na prática médica, com alta prevalência na população. A imensa maioria será diagnosticada e manejada na APS, pelo médico de família, que avaliará o tratamento inicial ou a necessidade de referenciar.

$\mathrm{Na}$ doença de graus I e II, há benefício de tratamentos clínicos conservadores que devem ser realizados no âmbito da APS, como o uso de fibras formadoras de massa e de flavonoides. (Fluxograma 1). 


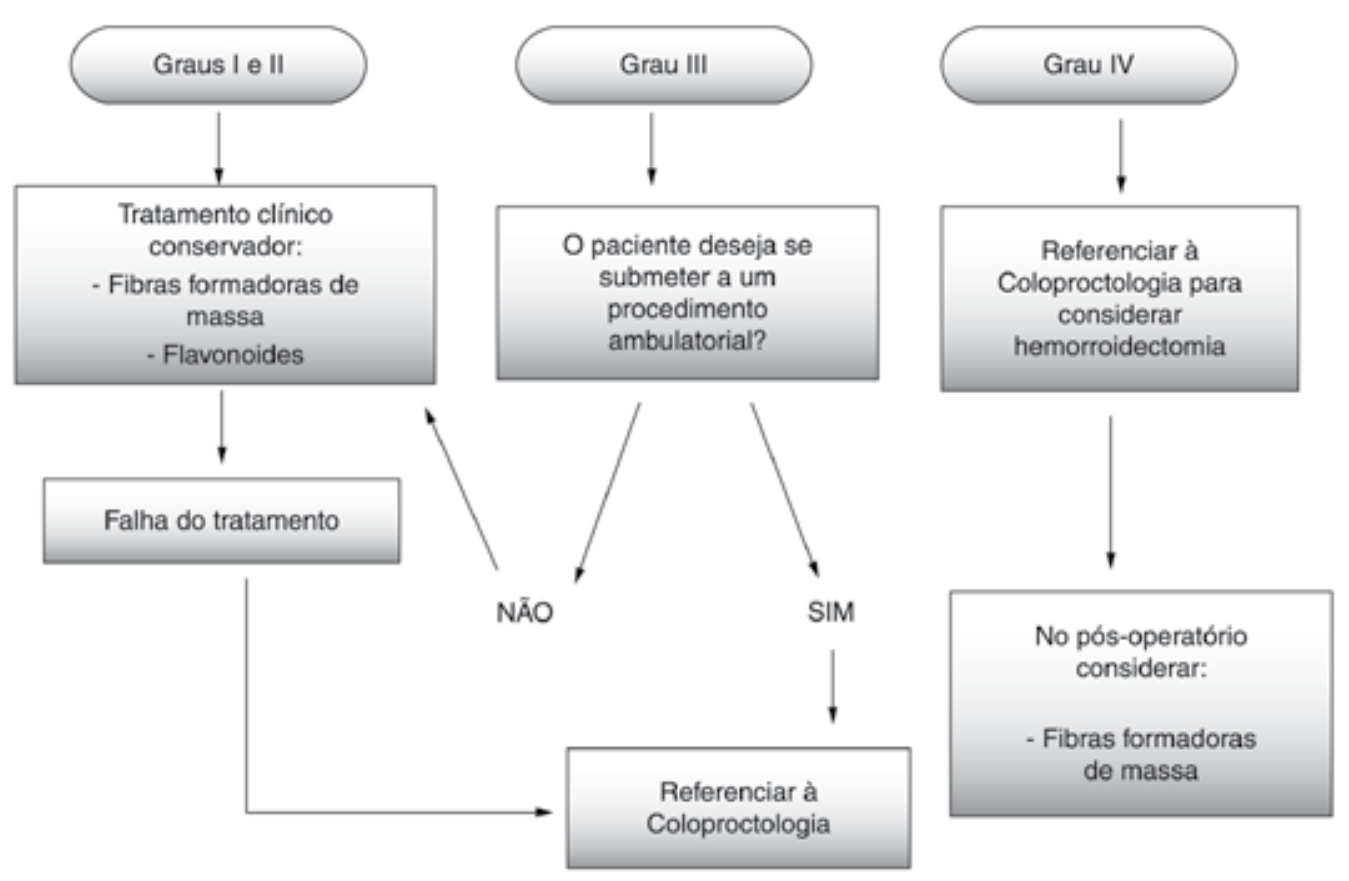

Fluxograma 1. Manejo da doença hemorroidária.

As fibras formadoras de massa minimizam a constipação e o esforço prolongado associado. Promovem a melhora global dos sintomas e do sangramento, sem diferença significante na incidência de efeitos adversos. Não há evidência de benefício para dor ou prurido como sintomas isolados. ${ }^{10}$ No mercado brasileiro, existem medicamentos à base de Plantago ovata e Plantago psyllium. A Relação de Medicamentos Essenciais (Rename) traz Plantago ovata para constipação e síndrome do intestino irritável, mas não menciona o uso para doença hemorroidária. A inclusão desta indicação poderia ser considerada numa próxima atualização da Rename.

Os flavonoides não têm seu mecanismo de ação bem estabelecido, mas estão associados ao fortalecimento da parede vascular e normalização da permeabilidade capilar. ${ }^{30}$ Demonstram benefício na melhora global dos sintomas, no sangramento, na exsudaçáo perianal e na recorrência dos sintomas após um episódio agudo, sem diferença na incidência de efeitos adversos (que em sua maioria correspondem a casos isolados de desconforto gastrointestinal). ${ }^{7,28}$ Seu uso por períodos maiores (em torno de 60 dias ou mais) parece mostrar efeitos ainda melhores se comparados a curtos períodos de seguimento. ${ }^{28}$ Para dor e prurido, ainda há controvérsia entre os estudos e não se pode confirmar a existência de benefício. No mercado brasileiro, estâo disponíveis medicamentos com rutosídeo e com associação de diosmina e hesperidina. Troxerrutina só existe em associaçôes não avaliadas nos ensaios clínicos. Nenhum destes medicamentos faz parte da Rename.

Em pessoas com doença hemorroidária grau III, que não desejam se submeter a um procedimento ambulatorial, pode-se optar pelo uso de fibras formadoras de massa ou flavonoides, já que há beneficio no que concerne à melhora global dos sintomas e sangramento. No entanto não há diferença significante no prolapso. ${ }^{7,28}$ Quando não ocorre melhora com o tratamento clínico conservador e na doença grau IV, a melhor conduta do médico na APS seria o encaminhamento à atenção secundária, para a especialidade de coloproctologia.

Casos submetidos a procedimentos ambulatoriais ou hemorroidectomia retornam à APS e, quando sintomáticos, podem se beneficiar do uso de fibras formadoras de massa, que diminuem a recorrência dos sintomas após ligadura elástica, ${ }^{28}$ ou de flavonoides, que reduzem o risco de sangramento em ambos os cenários. ${ }^{7}$

Para gestantes portadoras de doença hemorroidária graus I e II, o uso de flavonoides do tipo rutosídeo apresenta benefício na melhora global dos sintomas em quatro semanas, sem diferença estatística do placebo na ocorrência de efeitos adversos ou prejudiciais ao bebê. No entanto, o pequeno tamanho da amostra dos ensaios torna os dados insuficientes para afirmar a segurança de tais medicamentos na gestação. ${ }^{12}$

Intervençôes como analgésicos orais e tópicos, corticosteroides tópicos, banhos de assento e pomadas à base de nitroglicerina são amplamente utilizados e prescritos na APS, com aparentes efeitos benéficos. Todavia, não há evidências consistentes que validem seu uso. 


\section{Conclusão}

A maioria dos estudos incluídos nas meta-análises foi considerada de qualidade moderada, com problemas diversos como sigilo de alocação, cegamento, tempo curto e perda de seguimento e análise por protocolo. Não se pode descartar a ocorrência de viés de publicação, embora os autores tenham procurado minimizá-lo. Ensaios clínicos controlados e aleatorizados com adequada qualidade metodológica e maior tempo de seguimento são necessários para confirmar os resultados encontrados com fibras e flavonoides e para avaliar se existe benefício com outros tratamentos clínicos conservadores na doença hemorroidária.

\section{Referências}

1. Quilici FA, Reis Neto JA, Cordeiro F, Reis Junior JA, Ciquini S, editors. Atlas de proctologia: do diagnóstico ao tratamento. São Paulo: Lemos Editorial; 2000.

2. Cruz GMG. Coloproctologia: propedêutica nosológica. Rio de Janeiro: Revinter; 1999. (Coloproctologia, vol. II).

3. Cruz GMG. Coloproctologia: terapêutica. Rio de Janeiro: Revinter; 2000. (Coloproctologia, vol. III).

4. Loder PB, Kamm MA, Nicholls RJ, Phillips RK. Haemorrhoids: pathology, pathophysiology and aetiology. Br J Surg. 1994;81(7):946-954. http://dx.doi.org/10.1002/bjs.1800810707

5. Beck DE. Hemorrhoidal disease. In: Beck DE, Wexner SD, editors. Fundamentals of anorectal surgery. 2n ed. London: WB Saunders; 1998. p. 237-253.

6. Cruz GMG, Ferreira RMRS, Neves PM. Doença hemorroidária: aspectos epidemiológicos e diagnósticos de 9.289 pacientes portadores de doença hemorroidária. Rev Bras Coloproct. 2006;26(1):6-23. http://dx.doi.org/10.1590/S0101-98802006000100001

7. Perera N, Liolitsa D, lype S, Croxford A, Yassin M, Lang P, et al. Phlebotonics for haemorrhoids. Cochrane Database Syst Rev. 2012;(8):CD004322. http://dx.doi.org/10.1002/14651858.CD004322.pub3

8. Abramowitz L, Godeberge P, Staumont G, Soudan D. Clinical practice guidelines for the treatment of hemorrhoid disease. Gastroen Clin Biol. 2001;25(6-7):674-702. Pmid:11673735.

9. Johanson JF, Sonnenberg A. The prevalence of hemorrhoids and chronic constipation: an epidemiologic study. J Gastro. 1990;98(2):380-386.

10. Alonso-Coello P, Guyatt G, Heels-Ansdell D, Johanson JF, Lopez-Yarto M, Mills E, et al. Laxatives for the treatment of hemorrhoids. Cochrane Database Syst Rev. 2005;(4):CD004649. http://dx.doi.org/10.1002/14651858.CD004649.pub2

11. Quijano CE, Abalos E. Conservative management of symptomatic and/or complicated haemorrhoids in pregnancy and the puerperium. Cochrane Database Syst Rev. 2005;(3):CD004077. http://dx.doi.org/10.1002/14651858.CD004077.pub2

12. Banov L, Knoepp LF, Erdman LH, Alia RT. Management of hemorrhoidal disease. J S C Med Assoc. 1985;7:398-401.

13. Quijano CE, Abalos E. Conservative management of symptomatic and/or complicated haemorrhoids in pregnancy and the puerperium. Cochrane Database Syst Rev. 2005;(3):CD004077. http://dx.doi.org/10.1002/14651858.CD004077.pub2

14. Johanson JF, Rimm A. Optimal nonsurgical treatment of hemorrhoids: a comparative analysis of infrared coagulation, rubber band ligation, and injection sclerotherapy. Am J Gastroenterol. 1992;87:1600-1606. Pmid:1442682.

15. Alonso-Coello P, Marzo M. Office evaluation and treatment of hemorrhoids. J Fam Pract. 2003;52(5):366-374. Pmid:12737769.

16. Bleday R, Breen E. Treatment of hemorrhoids. Waltham: UpToDate; 2012 [acesso em 2012 Oct 02]. Disponível em: http://www.uptodate.com/contents/treatment-of-hemorrhoids?source=search_result\&search=hemorrhoids\&selectedTitle=1 74\#H2

17. Prodigy. Haemorrhoids. Prodigy; 2012 [acesso em 2012 Oct 02]. Disponível em: http://prodigy.clarity.co.uk/topic/haemorrhoids.

18. American Gastroenterological Association (AGA). American Gastroenterological Association Medical Position Statement: diagnosis and treatment of hemorrhoids. J Gastro. 2004;126:1461-1462. http://dx.doi.org/10.1053/j.gastro.2004.03.001

19. McPhee SJ, Papadakis MA, Rabow MW. 2012 Current Medical Diagnosis \& Treatment. 51th ed. McGrawHill Medical; 2012.

20. Rivadeneira DE, Steele SR, Ternent C, Chalasani S, Buie WD, Rafferty JL. Standards practice task force of the American Society of Colon and Rectal Surgeons. Practice parameters for the management of hemorrhoids (revised 2010). Dis Colon Rectum. 2011;54(9):1059-1064. http://dx.doi.org/10.1097/DCR.0b013e318225513d

21. Conselho Nacional de Secretários de Saúde (BR). Assistência de média e alta complexidade no SUS. Brasília: CONASS; 2007.

22. Ministério da Saúde (BR). Média e alta complexidade. Brasília: Ministério da Saúde; 2003 [acesso em 2002 Set 28]. Disponível em: http://portal.saude.gov.br/portal/sas/mac/area.cfm?id_area=821.

23. Ministério da Saúde (BR). Portaria GM/MS n 648, de 28 de março de 2006. Aprova a Política Nacional de Atenção Básica, estabelecendo a revisão de diretrizes e normas para a organização da Atenção Básica para o Programa Saúde da Família (PSF) e o Programa Agentes Comunitários de Saúde (PACS). Brasília: Ministério da Saúde; 2006. [acesso em 2012 Sep 28]. Disponível em: http://bvsms.saude.gov.br/bvs/publicacoes/prtGM648_20060328.pdf. 
24. Ministério da Saúde (BR), Secretaria de Atenção à Saúde, Departamento de Atenção Básica. Política Nacional de atenção básica. $4^{a}$ ed. Brasília: Ministério da Saúde; 2007. 68 p. (Série E. Legislação de Saúde. Série Pactos pela Saúde 2006, vol. 4).

25. Giovanella L, Mendonça MHM. Atenção primária à saúde. In: Giovanella L, Escorel S, Lobato LVC, Noronha JC, Carvalho Al. Políticas e sistema de saúde no Brasil. Rio de Janeiro: Fiocruz; 2008. p. 575-590.

26. Göttems LBD, Pires MRGM. Para além da atenção básica: reorganização do SUS por meio da interseção do setor político com o econômico. Saúde Soc. 2009;18(2):189-198 [acesso em 2012 Set 28]. http://dx.doi.org/10.1590/S0104-12902009000200003

27. Ministério da Saúde (BR); Secretaria de Ciência, Tecnologia e Insumos Estratégicos; Departamento de Ciência e Tecnologia. Diretrizes metodológicas: elaboração de pareceres técnico-científicos. $3^{\mathrm{a}}$ ed. Brasília: Ministério da Saúde; 2011.

28. Sackett DL, Richardson WS, Rosenberg W, Haynes RB. Evidence-Based Medicine: how to practice and teach EBM. 2th ed. London: Churchill Livingstone; 1997.

29. Alonso-Coello P, Zhou Q, Martinez-Zapata MJ, Mills E, Heels-Ansdell D, Johanson JF, et al. Meta-analysis of flavonoids for the treatment of haemorrhoids. Br J Surg. 2006;93:909-920. http://dx.doi.org/10.1002/bjs.5378

30. Cundal JD, Gunn J, Tilsed JVT, Duthie GS. The dose response of the internal anal sphincter to topical application of glyceryl trinitrate ointment. Colorectal Dis. 2001;3(4):259-262. http://dx.doi.org/10.1046/j.1463-1318.2001.00248.x

31. Beck DE, Roberts PL, Saclarides TJ, Senagore AJ, Stamos MJ, Wexner SD, editors. The American Society of Colon and Rectal Surgeons Textbook of Colon and Rectal Surgery. 2th ed. Springer; 2011. http://dx.doi.org/10.1007/978-1-4419-1584-9 